\section{Renovation of the Matrix Model of Glycerolipid Molecular Species}

Koretaro Takahashi*1 and Makoto Egi*2

(Accepted August 15, 1986)

The matrix model of glycerolipid ${ }^{15}$ that has been expressed as parallel lines on a semilogarithmic graph was reviewed because of the following reasons:

1. Though the proposed matrix model, in theory, can discerned the binding position of the fatty acid residue, the data of the $\alpha, \beta$ isomers were actually insufficient.

2. The scale of the vertical axis i.e. the logarithm of the relative retention time (RRT) was too short on the semilogarithmic graph.

Fortunately, the latest work done by Takamura et al. ${ }^{2)}$ gave valuable informations of RRTs of the $\alpha, \beta$ discerned molecular species of glycerolipids. So the RRT data of Takamura et al. ${ }^{2)}$ were used in this study. In addition, the scale of the vertical axis of the semilogarithmic graph was magnified in this study in order to gain a further insight into the matrix model of glycerolipids.

Fig. 1 illustrates the semilogarithmic plots of RRT's against total acyl carbon number's (CN's) or number of total double bond's (DB's). Lines from $A_{1}^{\alpha}$ to $A_{10}{ }^{\alpha}$ demonstrate the linking of the molecular species that differ only in the acyl carbon numbers which are 16 and 18. These lines seem to be parallel to each other, though $\mathrm{A}_{4}{ }^{\beta}$ exhibited a slightly gentle slope presumably owing to the differences in binding position of the acyl group. Lines from $B_{1}^{\beta}$ to $B_{5}{ }^{\beta}$ demonstrate the linking of the molecular species that differ only in the acyl carbon numbers which are 20 and 22 . These lines also seem to be parallel to each other. It was considered in the previous paper') that all the oblique lines on the semilogarithmic graph of RRT's versus CN's are parallel to each other regardless of carbon chain length or binding position of the acyl group. But this figure shows that the slope of the long chain (the " $\mathrm{B}$ " lines) is always gentle compared with the short one (the " $A$ " lines). The repugnance between the previous results ${ }^{1 / 2}$ and the results obtained in this study can be explained as follows: In the previous report, ${ }^{1)}$ the scale of the vertical axis, the logarithm of the RRT, was too short to recognize the differences in the slopes of the oblique lines between the long chain and the short one so that all the lines seemed to be parallel regardless of the carbon chain length. The results obtained in this study show that although there is a proximate parallel correlations among all lines on the semilogarithmic plots of RRT's versus CN's, another factor might contribute on the slope in accordance with the elongation of the acyl carbon chain. One elucidation of this aspect might be as follows: As it is illustrated, the slopes of the " $F$ " lines i.e. the linking of the molecular species that have the penta and hexaene acyl groups are always gentle, followed by the " $\mathrm{E}$ " lines (tetra and pentaene), the " $D$ " lines (mono and diene) and the " $C$ " lines (saturated and monoene). Therefore, the penta and the hexaene acyl groups that in most of the cases are the fatty acid residues of the 20 and 22 carbon chains, exhibit a more gentle slope compared with the 16 and 18 acyl carbon chains that are usually the saturated or

*1 Laboratory of Food Chemistry I, Faculty of Fisheries, Hokkaido University, Hakodate 041, Japan (高橋是太郎: 北海道大学水産学部).

*2 Kyowa Hakko Kogyo, Machida, Tokyo 194, Japan (江木 表：協和発醇工業).

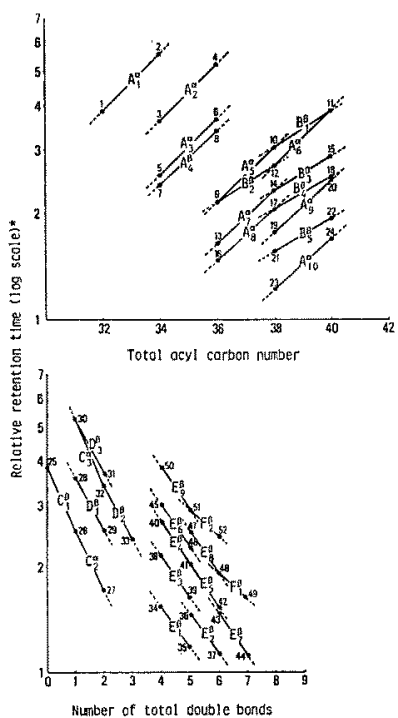

Fig. 1. Magnified RRT against CN or DB on HPLC. Data from Takamura et al. ${ }^{2)}$

* Retention time of di-12: 0 is regarded as 1.00.

The Greek letter superscript on the alphabet shows the acyl carbon number or number of double bonds substituted position.

$A_{1}^{\alpha}, A_{2}^{\alpha}, A_{3}^{\alpha}, A_{4}^{\alpha}, A_{5}{ }^{\alpha}, A_{8}{ }^{\alpha}, A_{7}{ }^{\alpha}, A_{8}{ }^{\alpha}, A_{8}{ }^{\alpha}, A_{10}{ }^{\alpha}$; Linking between the molecular species that differ only in the acyl carbon number which are 16 and 18.

$\mathbf{B}_{1}^{\beta}, \mathbf{B}_{2}{ }^{\beta}, \mathbf{B}_{3}{ }^{\beta}, \mathbf{B}_{4}{ }^{\beta}, \mathbf{B}_{5}{ }^{\beta}$ : Ditto 20 and 22.

$\mathrm{C}_{1} \beta, \mathrm{C}_{2}^{\alpha}, \mathrm{C}_{3}^{\alpha}$ : Linking between the molecular species that differ only in the acyl double bonds which are 0 (saturated) and 1 (monoene).

$\mathrm{D}_{1}{ }^{\beta}, \mathrm{D}_{2}{ }^{\beta}, \mathrm{D}_{3}{ }^{\beta}$ : Ditto 1 and 2 .

$\mathrm{E}_{1}^{\beta}, \mathrm{E}_{2}^{\beta}, \mathrm{E}_{8}^{\beta}, \mathrm{E}_{4}^{\beta}, \mathrm{E}_{8}^{\beta}, \mathrm{E}_{8}{ }^{\beta}, \mathrm{E}_{7}^{\beta}, \mathrm{E}_{8}{ }^{\beta}, \mathrm{E}_{8}{ }^{\beta}$ : Ditto 4 and 5.

$\mathrm{F}_{1}^{\beta}, \mathrm{F}_{8}{ }^{\beta}:$ Ditto 5 and 6.

Lines with the same alphabet are parallel.

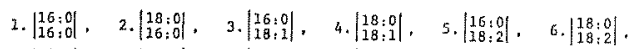

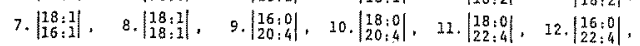

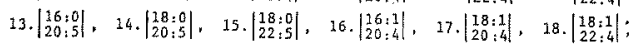

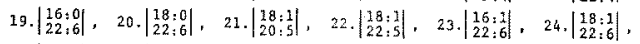
25. $\left|\begin{array}{l}16: 0 \\ 16: 0\end{array}\right|, 25 \cdot\left|\begin{array}{l}16: 0 \\ 16: 1\end{array}\right|, 27 \cdot\left|\begin{array}{l}16: 1 \\ 16: 1\end{array}\right|, 28 \cdot\left|\begin{array}{l}16: 0 \\ 18: 1\end{array}\right|, 29 \cdot\left|\begin{array}{l}16: 0 \\ 18: 2\end{array}\right|, 30 \cdot\left|\begin{array}{l}18: 0 \\ 18: 1\end{array}\right|$, $31 .\left|\begin{array}{l}18: 0 \\ 18: 2\end{array}\right|, 32\left|\begin{array}{c}18: 3 \\ 18: 1\end{array}\right|, 33 .\left|\begin{array}{l}18: 1 \\ 18: 2\end{array}\right|, 34 \cdot\left|\begin{array}{l}14: 0 \\ 20: 4\end{array}\right|, 35 \cdot\left|\begin{array}{l}14: 0 \\ 20: 5\end{array}\right|, 36 \cdot\left|\begin{array}{l}16: 1 \\ 20: 4\end{array}\right|$, $37 \cdot\left|\begin{array}{l}16: 5 \\ 20: 5\end{array}\right|, 38 \cdot\left|\begin{array}{l}16: 0 \\ 20: 4\end{array}\right|, 39 \cdot\left|\begin{array}{l}16: 0 \\ 20: 5\end{array}\right|, 40 \cdot\left|\begin{array}{c}16: 0 \\ 22: 4\end{array}\right|, 41 . \mid\left[\begin{array}{l}16: 9 \\ 22,5\end{array} \mid\right.$ and $\left|\begin{array}{l}18: 1 \\ 20: 4\end{array}\right|$, $42 .\left|\begin{array}{ll}18: 1 \\ 20: 5\end{array}\right|, 43 \cdot\left|\begin{array}{c}18: 2 \\ 20: 4\end{array}\right|, 44 \cdot\left|\begin{array}{l}18: 2 \\ 20: 5\end{array}\right|, 45 \cdot\left|\begin{array}{l}18: 0 \\ 20: 4\end{array}\right|, 46,\left|\begin{array}{l}18: 0 \\ 20: 5\end{array}\right|, 47 .\left|\begin{array}{l}18: 1 \\ 22: 4\end{array}\right|$, 48. $\left|\begin{array}{l}18: 1 \\ 22: 5\end{array}\right|, 49 \cdot\left|\begin{array}{l}18: 1 \\ 22: 6\end{array}\right|, 50 \cdot\left|\begin{array}{l}18: 0 \\ 22: 4\end{array}\right|, 51 \cdot\left|\begin{array}{l}18: 0 \\ 22 ; 5\end{array}\right|, 52 \cdot\left|\begin{array}{l}18: 0 \\ 22 ; 6\end{array}\right|$.

$\alpha, \beta$ isomers are all discerned here.

the monoene fatty acid residues.

The matrix model of glycerolipid should be renovated to some extent from the aforementioned aspect.

\section{References}

1) K. Takahashi: Mem. Fac. Fish. Hokkaido Univ., 32, 245-330 (1985).

2) H. Takamura, H. Narita, R. Urade, and M. Kito: Lipids, 21, 356-361 (1986). 